\title{
Message from the General Chair
}

welcome you to the 2015 IEEE International Conference on Microelectronic Systems Education (MSE '15). Since its inception in 1997, the goal of the MSE conference has been to provide a venue where university educators with an interest in the design, implementation, and testing of microelectronic systems can share their experiences, learn about best practices, and explore future trends in both technology and educational methods. MSE is held during odd years in coordination with our European partner conference the European Workshop on Microelectronics Education (EWME), which is held in even years.

With the $10^{\text {th }}$ meeting of this conference we can reflect on the challenges and opportunities for microelectronics systems designers and educators. Thanks to the fruits of 50 years of Moore's Law and many other technological advances, microelectronic systems have never been more ubiquitous, or paradoxically, more invisible - a trend that can only accelerate as the Internet of Things becomes a reality. Microelectronics educators need to help students see inside the "black box" of technology and share in the excitement of creating new innovations. At the same time, we need to train them to design and bring to market increasingly complex systems that can operate safely and securely in potentially hostile environments. Program Chair Ozcan Ozturk has led the program committee in putting together an exciting program that focuses on these challenges and opportunities.

The $10^{\text {th }}$ meeting of MSE also marks a new direction for the conference as we co-locate for the first time with the Great Lakes Symposium on VLSI (GLSVLSI). The MSE Organizing Committee felt that this move would increase opportunities to attract participants and authors, and we are pleased to see an increase in submitted papers this year as a result. We thank the leadership of GLVLSI for welcoming us in Pittsburgh.

The continuing success of the MSE Conference is due to the tireless efforts of many people. I would particularly like to acknowledge the contributions of the program committee under the leadership of Program Chair Ozcan Ozturk in putting together an exciting program and the work by many members of the Organizing Committee for handling the logistical details of the conference. I would particularly like to thank Don Bouldin (Steering Committee Chair) for his continued guidance of MSE and for stepping up to superbly handle local arrangements yet again. Finally, I would like to acknowledge the financial support of our industrial patrons which has helped make this conference possible.

I would like to close by inviting our current authors and participants to get further involved with the MSE Conference. There are many opportunities to contribute to this conference on both the Program Committee and Organizing Committee, and I think that you will find your involvement very rewarding as your leadership sets the future direction of the conference. If you are interested, please don't hesitate to talk to me or any other member of the MSE leadership. In the meantime, enjoy the conference and Pittsburgh.

\author{
General Chair \\ John A. Nestor \\ Lafayette College
}




\section{Message from the Program Chair}

W elcome to the 2015 IEEE International Conference on Microelectronic Systems Education (MSE'15). Our conference program includes 14 papers contributed by microelectronics educators and practitioners from around the world. These papers were selected from submitted manuscripts after rigorous review by our Program Committee.

The MSE Conference has a tradition of encouraging lots of discussion among its attendees with its single-track conference and large poster sessions. This year, to foster these discussions, the program committee has invited each of the 12 papers selected for oral presentation to also provide poster presentations. In this manner, there will be more opportunities for discussions among the authors and others attending the conference.

The theme of this year's conference is "Microelectronics Education in the Modern Era". With the rapidly shifting requirements of microelectronic systems due to challenges associated with Moore's law, microelectronics education needs to adapt. This year's program includes a panel of industry and academic experts to discuss the problems and solutions to this dilemma.

Our Keynote Speaker is Donald E. Thomas, Professor of Electrical and Computer Engineering at Carnegie Mellon University, working in the areas of single-chip heterogeneous multiprocessor systems, FPGA acceleration of computational tasks, and chip architecture design for yield improvement and lifetime reliability. Prof. Thomas will open the conference's theme with a talk on "Moving Digital System Design Courses into the Modern Era, Again" in which he addresses his experiences in Sophomore, Junior, and Senior level logic design courses.

I would like to conclude by thanking our Program Committee for their efforts to rigorously review papers and ensure the high quality of our technical program, and the Organizing Committee for their efforts in putting together the entire conference. The steering committee provided valuable guidance on interpreting and setting policies. The organizing committee and the general chair, John Nestor, deserve special thanks for taking care of all the logistics and allowing me to focus on the technical program, and for bringing us together for the conference. I also would like to thank Proceedings Chair Tina Hudson for her help in taking the technical program from the initial call for papers to the assembled proceedings. Finally, I would like to thank Steering Committee Chair Don Bouldin for giving me the opportunity to serve as Program Chair and for all of their efforts to make this conference a success.

\section{Program Chair \\ Ozcan Ozturk \\ Bilkent University}

